\title{
Trainable COSFIRE filters for vessel delineation with application to retinal images
}

\author{
George Azzopardi ${ }^{a}$, Nicola Strisciuglio ${ }^{\mathrm{a}, \mathrm{b}, *}$, Mario Vento $^{\mathrm{b}}$, Nicolai Petkov ${ }^{\mathrm{a}}$ \\ a Johann Bernoulli Institute for Mathematics and Computer Science, University of Groningen, The Netherlands \\ ${ }^{\mathrm{b}}$ Dept. of Information and Electrical Engineering and Applied Mathematics (DIEM), University of Salerno, Italy
}

\section{A R T I C L E I N F O}

\section{Article history:}

Received 7 October 2013

Received in revised form 11 April 2014

Accepted 26 August 2014

Available online 3 September 2014

\section{Keywords:}

COSFIRE

Delineation

Retinal image analysis

Trainable filters

Vessel segmentation

\begin{abstract}
A B S T R A C T
Retinal imaging provides a non-invasive opportunity for the diagnosis of several medical pathologies. The automatic segmentation of the vessel tree is an important pre-processing step which facilitates subsequent automatic processes that contribute to such diagnosis.

We introduce a novel method for the automatic segmentation of vessel trees in retinal fundus images. We propose a filter that selectively responds to vessels and that we call $B$-COSFIRE with $B$ standing for bar which is an abstraction for a vessel. It is based on the existing COSFIRE (Combination Of Shifted Filter Responses) approach. A B-COSFIRE filter achieves orientation selectivity by computing the weighted geometric mean of the output of a pool of Difference-of-Gaussians filters, whose supports are aligned in a collinear manner. It achieves rotation invariance efficiently by simple shifting operations. The proposed filter is versatile as its selectivity is determined from any given vessel-like prototype pattern in an automatic configuration process. We configure two B-COSFIRE filters, namely symmetric and asymmetric, that are selective for bars and bar-endings, respectively. We achieve vessel segmentation by summing up the responses of the two rotation-invariant $B$-COSFIRE filters followed by thresholding.

The results that we achieve on three publicly available data sets (DRIVE: $\mathrm{Se}=0.7655, \mathrm{Sp}=0.9704$; STARE: $S e=0.7716, S p=0.9701$; CHASE_DB1: $S e=0.7585, S p=0.9587)$ are higher than many of the state-of-the-art methods. The proposed segmentation approach is also very efficient with a time complexity that is significantly lower than existing methods.
\end{abstract}

(c) 2014 Elsevier B.V. All rights reserved.

\section{Introduction}

Retinal fundus images (Fig. 1a) are routinely used for the diagnosis of various pathologies, including age-related macular degeneration and diabetic retinopathy - the two leading causes of blindness among people of the Western World (Abramoff et al., 2010) - as well as glaucoma, hypertension, arteriosclerosis and multiple sclerosis.

The computer analysis of retinal fundus images is an alternative to direct ophthalmoscopy where a medical specialist visually inspects the fundus of the retina. Although ophthalmoscopy provides an effective means of analysing the retina, there is evidence that fundus photographs are more reliable than ophthalmoscopy, for instance, in the diagnosis of diabetic retinal lesions (Harding et al., 1995; von Wendt et al., 1999). Moreover, retinal fundus photography provides the possibility of analysing the produced images in batch mode. The manual analysis of retinal images is, on the

\footnotetext{
* Corresponding author at: Johann Bernoulli Institute for Mathematics and Computer Science, University of Groningen, The Netherlands.
}

other hand, time-consuming and expensive. The automation of certain processing steps is thus important and facilitates the subsequent decisions by specialists to provide a basis for further automatic steps in the early diagnosis of specific diseases.

The automatic segmentation of blood vessels from background (Fig. 1b) is one of the basic steps that is required for the analysis of retinal fundus images. This is a challenging process mainly due to the width variability of vessels and due to the low quality of retinal images that typically contain noise and changes of brightness. Several methods have already been proposed for the segmentation of blood vessels in such images, which can be divided into the following two categories: unsupervised and supervised methods. Supervised methods use pixel-wise feature vectors to train a classifier in order to discriminate between vessel and non-vessel pixels, while unsupervised methods do not use classifiers but rely on thresholding filter responses or other rule-based techniques.

Vessel tracking techniques (Liu and Sun, 1993; Zhou et al., 1994; Chutatape et al., 1998; Tolias and Panas, 1998) are unsupervised methods that use an initial set of points, which are chosen either manually or automatically, to obtain the vascular tree by 


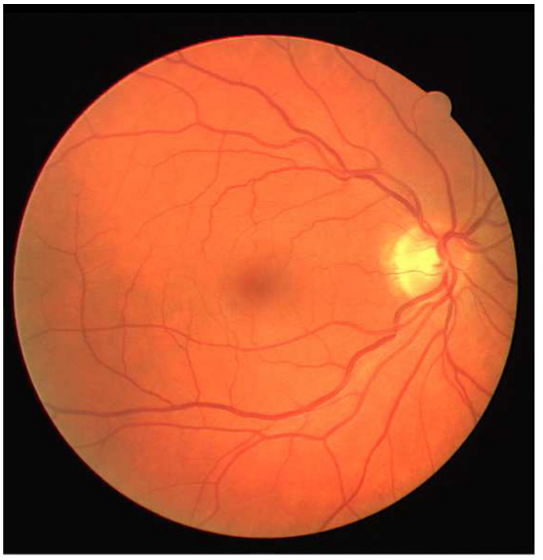

(a)

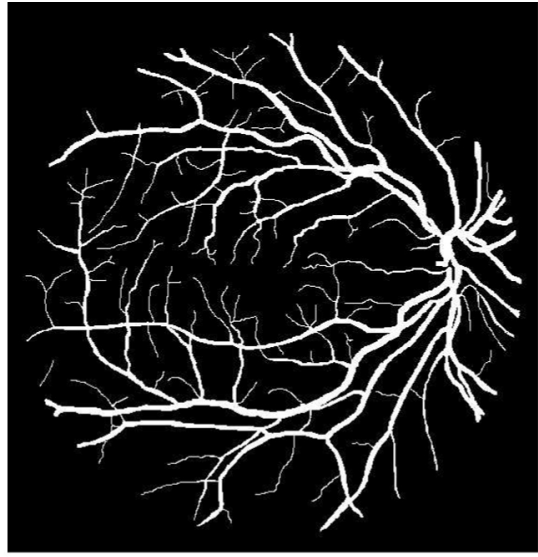

(b)

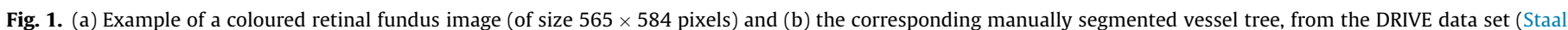
et al., 2004). (For interpretation of the references to colour in this figure legend, the reader is referred to the web version of this article.)

following the vessel center lines. Other unsupervised methods, which use a priori information about the profile structure of the vessels, employ mathematical morphology to segment the vessel tree from the background (Zana and Klein, 2001; Heneghan et al., 2002; Mendonca and Campilho, 2006). Moreover, morphological operators have been used to enhance the vessels and then combine curvature analysis and linear filtering to discriminate the vessels from the background (Fang et al., 2003). Matched filtering techniques (Chauduri et al., 1989; Hoover et al., 2000; Gang et al., 2002; Al-Rawi et al., 2007) model the profile of the vessels by using a two-dimensional (2D) kernel with a Gaussian cross-section. Growing a "Ribbon of Twins" active contour model has been used both for segmentation and for width measurement of the vessels (Al-Diri et al., 2009). Martinez-Pérez et al. (2007) proposed a method based on multiscale analysis to obtain vessels width, size and orientation information that are used to segment the vessels by means of a growing procedure. Lam et al. (2010) proposed a multiconcavity modeling approach with differentiable concavity measure to handle both healthy and unhealthy retinal images simultaneously.

On the other hand, supervised methods have been used to automatically label pixels either vessel or non-vessel. In such methods, classifiers are trained by pixel-wise feature vectors that are extracted from training retinal images, whose ground truth labels are given in the corresponding manually labeled images. For instance, a k-Nearest Neighbor (kNN) approach was used by Niemeijer et al. (2004) and Staal et al. (2004) to classify the feature vectors that were constructed by a multiscale Gaussian filter and by a ridge detector, respectively. Soares et al. (2006) used a Bayesian classifier in combination with multiscale analysis of Gabor wavelets. A multilayer neural network was applied by Marin et al. (2011) to classify pixels based on moment-invariant features. Ricci and Perfetti (2007) proposed a rotation-invariant line operator both as an unsupervised method as well as in combination to a support vector machine (SVM) with a linear kernel. Fraz et al. (2012) employed a classification scheme based on an ensemble of boosted and bagged decision trees.

Most of the existing unsupervised methods are based on filtering techniques that rely on linear operations using predefined kernels. In particular, the output of those filtering methods is essentially a summation of weighted neighboring pixels (template matching). For instance, Al-Rawi et al. (2007) convolve (weighted sum) a pre-processed retinal image with second-derivative Gaussian kernels, and Ricci and Perfetti (2007) use a set of fixed hand-crafted templates. Template matching methods are sensitive to slight deformations from the expected pattern.
In this paper, we introduce a novel method for the automatic segmentation of blood vessels in retinal fundus images. It is based on the Combination of Receptive Fields (CORF) computational model of a simple cell in visual cortex (Azzopardi and Petkov, 2012) and its implementation called Combination of Shifted Filter Responses (COSFIRE) (Azzopardi and Petkov, 2013b). We propose a bar-selective COSFIRE filter, or B-COSFIRE for brevity, that can be effectively used to detect bar-shaped structures such as blood vessels. The B-COSFIRE filter that we propose is non-linear as it achieves orientation selectivity by multiplying the output of a group of Difference-of-Gaussians (DoG) filters, whose supports are aligned in a collinear manner. It is tolerant to rotation variations and to slight deformations. Moreover, unlike the handcrafted methods mentioned above, COSFIRE is a trainable filter approach. It means that the selectivity of the filter is not predefined in the implementation but it is determined from a user-specified prototype pattern (e.g. a straight vessel, a bifurcation or a crossover point) in an automatic configuration process.

We evaluated the proposed method on the following three publicly available data-sets: DRIVE (Staal et al., 2004), STARE (Hoover et al., 2000) and CHASE_DB1 (Owen et al., 2009). Other data sets, such as the REVIEWDB (Al-Diri et al., 2008) and BioImLab (Grisan et al., 2008) data sets have not been considered in the evaluation of our method as they are designed to evaluate vessels width measurement and tortuosity estimation algorithms, respectively.

The rest of the paper is organized as follows: in Section 2 we explain the proposed method and show how the $B$-COSFIRE filter can be configured to detect blood vessels. In Section 3 we evaluate our method. We provide a discussion in Section 4 and finally we draw conclusions in Section 5.

\section{Proposed method}

\subsection{Overview}

In the following, we explain how the proposed $B$-COSFIRE filter is configured and used for the segmentation of the vessel tree in a given retinal fundus image. Before we apply the $B$-COSFIRE filter, we perform a pre-processing step to enhance the contrast of the vessels and to smooth the border of the field-of-view (FOV) of the retina. We elaborate on this pre-processing aspect in Section 3.2. We also demonstrate how tolerance to rotation is achieved by simply manipulating some parameters of the model.

Fig. 2 illustrates the principle design of the proposed $B$-COSFIRE filter that is configured to be selective for a vertical bar. It uses as 

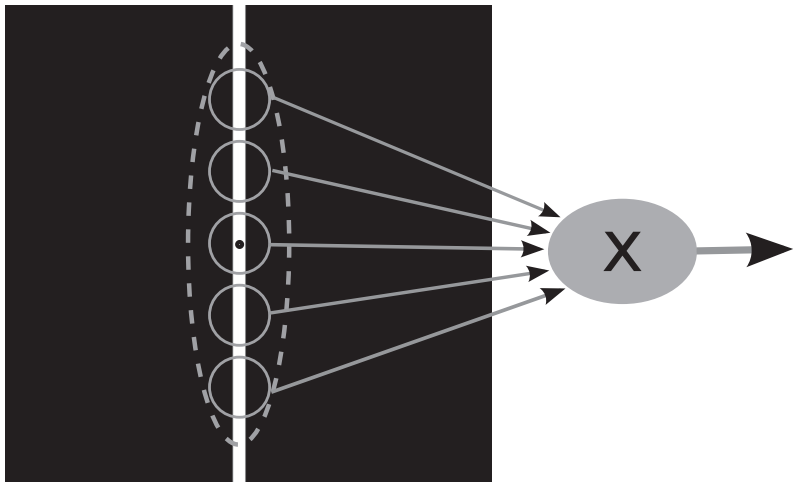

Fig. 2. Sketch of the proposed B-COSFIRE filter. The black spot in the middle of the white bar indicates the center of the filter support which is illustrated as a dashed ellipse. A $B$-COSFIRE filter combines the responses from a group of DoG filters (represented by the solid circles) by multiplication.

input the responses of center-on DoG filters at certain positions with respect to the center of its area of support. Such DoG filters give high responses to intensity changes in the input image. Each gray circle in Fig. 2 represents the area of support of a center-on DoG filter. The response of a $B$-COSFIRE filter is computed as the weighted geometric mean, essentially the product, of the responses of the concerned DoG filters in the centers of the corresponding circles. The positions at which we take their responses are determined by an automatic analysis procedure of the response of a DoG filter to a prototype bar structure; we explain this procedure below.

\subsection{Detection of changes in intensity}

We denote by $\operatorname{DoG}_{\sigma}(x, y)$ a center-on DoG function with an excitatory (i.e. positive) central region and an inhibitory (i.e. negative) surround:

$D o G_{\sigma}(x, y) \stackrel{\text { def }}{=} \frac{1}{2 \pi \sigma^{2}} \exp \left(-\frac{x^{2}+y^{2}}{2 \sigma^{2}}\right)-\frac{1}{2 \pi(0.5 \sigma)^{2}} \exp \left(-\frac{x^{2}+y^{2}}{2(0.5 \sigma)^{2}}\right)$

where $\sigma$ is the standard deviation of the Gaussian function that determines the extent of the surround. This type of function is an accepted computational model of some cells in the lateral geniculate nucleus (LGN) of the brain (Rodieck, 1965). Motivated by the results of electrophysiological studies of LGN cells in owl monkey (Irvine et al., 1993; Xu et al., 2002), we set the standard deviation of the inner Gaussian function to $0.5 \sigma$. For a given location $(x, y)$ and a given intensity distribution $I\left(x^{\prime}, y^{\prime}\right)$ of an image $I$, the response $c_{\sigma}(x, y)$ of a DoG filter with a kernel function $\operatorname{DoG}_{\sigma}\left(x-x^{\prime}, y-y^{\prime}\right)$ is computed by convolution:

$c_{\sigma}(x, y) \stackrel{\text { def }}{=}\left|I \star D o G_{\sigma}\right|^{+}$

where $\mid \cdot l^{+}$denotes half-wave rectification. ${ }^{1}$ Fig. $3 b$ shows the response image of a DoG filter that is applied to the synthetic input image shown in Fig. 3a.

\subsection{Configuration of a B-COSFIRE Filter}

Fig. 4 illustrates an example of the automatic configuration of a $B$-COSFIRE filter. We use a synthetic image that contains a vertical

${ }^{1}$ Half-wave rectification is an operation that suppresses (sets to 0 ) the negative values.

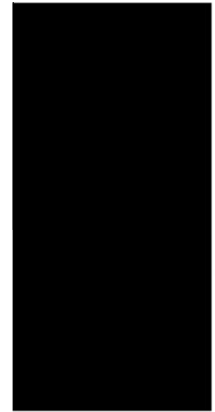

(a)

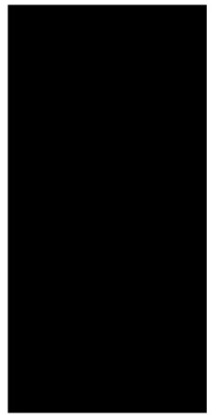

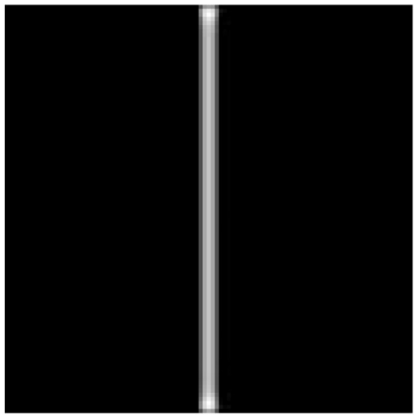

(b)
Fig. 3. (a) Synthetic input image (of size $100 \times 100$ pixels) of a vertical line $(5$ pixels wide) and (b) the corresponding response image of a center-on DoG filter (here $\sigma=2.6)$.

bar as prototype pattern, such as the one shown in Fig. 3a. We choose the center point, labeled by ' 1 ' in Fig. 4, and in an automatic process we analyse its local neighbourhood as described below.

We apply a center-on DoG filter with a given $\sigma$ to the prototype input pattern. We then consider the DoG filter responses $c_{\sigma}(x, y)$ along a number (in general $k$ ) of concentric circles around the center point (labelled by ' 1 ' in Fig. 4). The positions along these circles at which these responses reach significant local maxima are the positions of the points that characterize the dominant intensity variations around the point of interest. For the considered example, there are two such positions for each of the two circles. These points are labelled from ' 2 ' to ' 5 ' in Fig. 4 . The number of such points depends on the number $k$ of concentric circles we consider and the specified prototype pattern.

In the proposed $B$-COSFIRE filter, every point $i$ that is selected with the method mentioned above is described by a tuple of three parameters $\left(\sigma_{i}, \rho_{i}, \phi_{i}\right): \sigma_{i}$ represents the standard deviation of the DoG filter that responds most strongly and that provides the input, while $\rho_{i}$ and $\phi_{i}$ are the polar coordinates with respect to the center of support of the $B$-COSFIRE filter.

We denote by $S=\left\{\left(\sigma_{i}, \rho_{i}, \phi_{i}\right) \mid i=1, \ldots, n\right\}$ the set of 3-tuples of a $B$-COSFIRE filter, where $n$ stands for the number of considered DoG responses. In Eq. (3) we report the parameter values of a set $S$ that are determined by the automatic analysis of the input pattern shown in Fig. 4.

$S=\left\{\begin{array}{l}\left(\sigma_{1}=2.6, \rho_{1}=0, \phi_{1}=0\right), \\ \left(\sigma_{2}=2.6, \rho_{2}=2, \phi_{2}=1.57\right), \\ \left(\sigma_{3}=2.6, \rho_{3}=2, \phi_{3}=4.71\right), \\ \left(\sigma_{4}=2.6, \rho_{4}=4, \phi_{4}=1.57\right), \\ \left(\sigma_{5}=2.6, \rho_{5}=4, \phi_{5}=4.71\right)\end{array}\right\}$

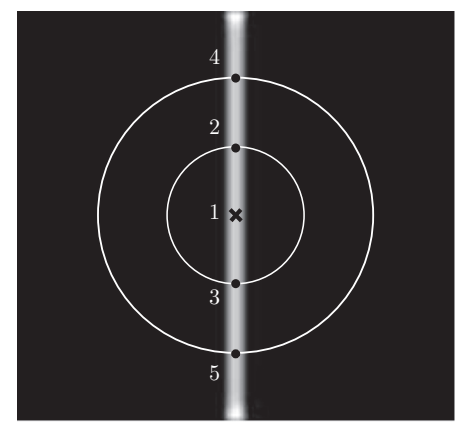

Fig. 4. Example of the configuration of a $B$-COSFIRE filter to be selective for a vertical bar. The center of the area of support is indicated by the cross marker. The enumerated spots represent the positions at which the strongest DoG responses are achieved along the concentric circles of given radii. 


\subsection{Blurring and shifting DoG responses}

The above configuration process results in a $B$-COSFIRE filter that is selective for the collinear spatial arrangement of five strong intensity variations. We use the DoG responses at the determined positions to compute the output of the B-COSFIRE filter.

First, we blur the DoG responses in order to allow for some tolerance in the position of the respective points. We define the blurring operation as the computation of the maximum value of the weighted thresholded responses of a DoG filter. For the weighting, we multiply the responses of the DoG filter by the coefficients of a Gaussian function $G_{\sigma^{\prime}}\left(x^{\prime}, y^{\prime}\right)$, whose standard deviation $\sigma^{\prime}$ is a linear function of the distance $\rho_{i}$ from the support center of the filter:

$\sigma^{\prime}=\sigma_{0}^{\prime}+\alpha \rho_{i}$

where $\sigma_{0}^{\prime}$ and $\alpha$ are constants.

Second, we shift each blurred DoG response by a distance $\rho_{i}$ in the direction opposite to $\phi_{i}$, so that they meet at the support center of the $B$-COSFIRE filter. The concerned shift vector is $\left(\Delta x_{i}, \Delta y_{i}\right)$ where $\Delta x_{i}=-\rho_{i} \cos \phi_{i}$ and $\Delta y_{i}=-\rho_{i} \sin \phi_{i}$.

We denote by $S_{\sigma_{i}, \rho_{i}, \phi_{i}}(x, y)$ the blurred and shifted response of a DoG filter for each tuple $\left(\sigma_{i}, \rho_{i}, \phi_{i}\right)$ in set $S$. Formally, we define the $i$-th blurred and shifted DoG response as:

$s_{\sigma_{i}, \rho_{i}, \phi_{i}}(x, y)=\max _{x^{\prime}, y^{\prime}}\left\{c_{\sigma_{i}}\left(x-\Delta x_{i}-x^{\prime}, y-\Delta y_{i}-y^{\prime}\right) G_{\sigma^{\prime}}\left(x^{\prime}, y^{\prime}\right)\right\}$,

where $-3 \sigma^{\prime} \leqslant x^{\prime}, y^{\prime} \leqslant 3 \sigma^{\prime}$.

\subsection{Response of a B-COSFIRE filter}

We define the output of a B-COSFIRE filter as the weighted geometric mean of all the blurred and shifted DoG responses that correspond to the tuples in the set $S$ :

$r_{S}(x, y) \stackrel{\text { def }}{=}\left|\left(\prod_{i=1}^{|S|}\left(s_{\sigma_{i}, \rho_{i}, \phi_{i}}(x, y)\right)^{\omega_{i}}\right)^{1 / \sum_{i=1}^{|S|} \omega_{i}}\right|_{t}$

$\omega_{i}=\exp ^{-\frac{\rho_{i}^{2}}{2 \tilde{\sigma}^{2}}}, \quad \hat{\sigma}=\frac{1}{3} \max _{i \in\{1 \ldots|S|\}}\left\{\rho_{i}\right\}$

where $|\cdot|_{t}$ stands for thresholding the response at a fraction $t(0 \leqslant t \leqslant 1)$ of the maximum responses. The weighted geometric mean is an AND-type function, that is a $B$-COSFIRE filter achieves a response only when all the afferent blurred and shifted responses $s_{\sigma_{i}, \rho_{i}, \phi_{i}}(x, y)$ are greater than zero. The contribution of the blurred and shifted responses decreases with an increasing distance from the center of the support of the B-COSFIRE filter. A B-COSFIRE filter is selective to a bar of a given preferred orientation, the one of the prototype bar structure that was used for its configuration.

\subsection{Achieving rotation invariance}

The orientation preference of a $B$-COSFIRE filter, as described above, depends on the orientation of the bar structure used as input for the configuration of the filter. One can configure a filter with a different orientation preference by presenting a rotated bar. Alternatively, one can manipulate the parameter in the set $S$, which corresponds to the orientation preference $0^{\circ}$, to obtain a new set $R_{\psi}(S)$ with orientation preference $\psi$ :

$R_{\psi}(S)=\left\{\left(\sigma_{i}, \rho_{i}, \phi_{i}+\psi\right) \mid \forall\left(\sigma_{i}, \rho_{i}, \phi_{i}\right) \in S\right\}$

In order to detect bars at multiple orientations, we merge the responses of $B$-COSFIRE filters with different orientation preferences by taking the maximum value at every location $(x, y)$ :

$\hat{r}_{S}(x, y) \stackrel{\text { def }}{=} \max _{\psi \in \Psi}\left\{r_{R_{\psi}(S)}(x, y)\right\}$ where $\Psi$ is a set of $n_{r}$ equidistant orientations given as $\Psi=\left\{\frac{\pi}{n_{r}} i \mid 0 \leqslant i<n_{r}\right\}$. Fig. 5c shows the response image of a rotation-invariant $B$-COSFIRE filter (12 values of $\psi: \psi=$ $\left.\left\{0, \frac{\pi}{12}, \frac{\pi}{6}, \ldots, \frac{11 \pi}{12}\right\}\right)$ to the retinal image shown in Fig. 5a. The images in Fig. $5 \mathrm{~d}-0$ show the response images of $B$-COSFIRE filters achieved for different $\psi$ values.

The computation of the output of a rotation-invariant $B$-COSFIRE filter is very efficient. It involves one convolution $(O(n \log n)$, where $n$ is the number of pixels in a given image) of the input image with a center-on DoG filter (Eq. (2)) followed by a maximum weighted operation (Eq. (5)) using a separable Gaussian filter $(O(k n)$, where $k$ is the number of pixels in a given kernel) for each of the unique values of the $\rho$ parameter. The response for each considered orientation is achieved by two linear-time $(O(n))$ operations: appropriate shifting of the pre-computed weighted DoG responses followed by weighted geometric mean. Finally, the rotation-invariant response is achieved by another linear-time operation that takes the pixel-wise maximum of all orientation response maps.

\subsection{Detection of bar endings}

The AND-type output function of a B-COSFIRE filter achieves a response only when all the afferent inputs are activated. In principle, a $B$-COSFIRE filter does not achieve a response at bar (or vessel) endings. In practice, however, due to noisy backgrounds in retinal images, a $B$-COSFIRE filter also achieves a response at the end of the vessels, but much lower than the response that is achieved in the middle of a vessel.

We address this matter by configuring a new $B$-COSFIRE filter by the prototype bar ending shown in Fig. 6a. Here, we use the point that lies on the end of the line as the point of interest. In order to distinguish between the two types of filters, we refer to this filter as asymmetric $B$-COSFIRE filter and to the previously defined filter as symmetric $B$-COSFIRE filter.

Fig. $7 \mathrm{~d}$ demonstrates that, in contrast to a symmetric $B$-COSFIRE filter, an asymmetric $B$-COSFIRE filter achieves much stronger response at the end of the vessel that is shown in Fig. $7 \mathrm{a}$.

\section{Results}

\subsection{Data sets and ground truth}

We use three publicly available data sets of retinal fundus images, called DRIVE (Staal et al., 2004), STARE (Hoover et al., 2000) and CHASE_DB1 (Owen et al., 2009). These data sets have gained particular popularity as they comprise the corresponding ground truth images that are manually segmented by different observers.

The DRIVE data set consists of 40 images (divided into a training set and a test set, each of which contains 20 images). For each image, the DRIVE data set contains a mask that delineates the FOV area together with the corresponding binary segmentation of the vessel tree. The images in the training set have been manually segmented by one human observer, while the images in the test set have been segmented by two other observers.

The STARE data set comprises 20 color retinal fundus images, 10 of which contain signs of pathologies. The data set contains two groups of manually segmented images prepared by two different observers.

The CHASE_DB1 data set contains 28 colour images of retinal fundus from 14 patients in the program Child Heart And Health Study in England. The data set contains two groups of manually segmented images provided by two observers.

For all the three data sets, the performance of the proposed method is measured by comparing the automatically generated 


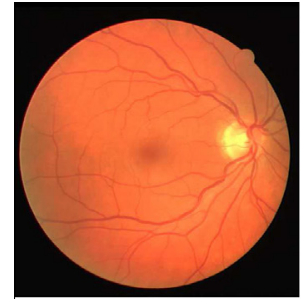

(a)

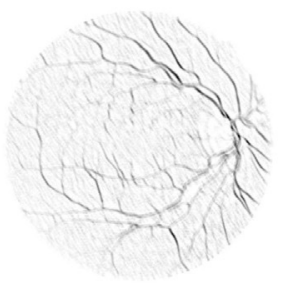

(f)

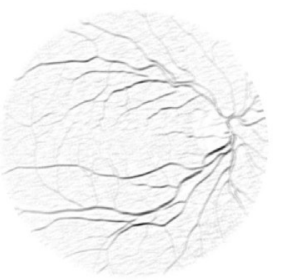

(k)

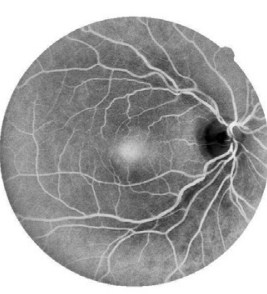

(b)

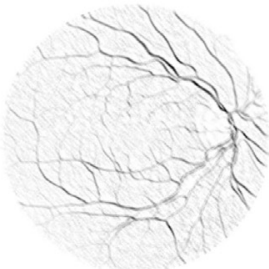

(g)

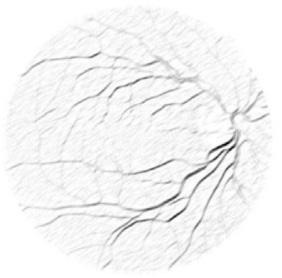

(1)

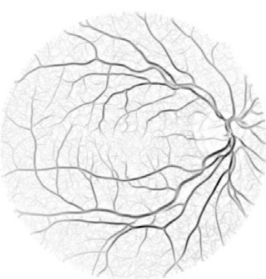

(c)

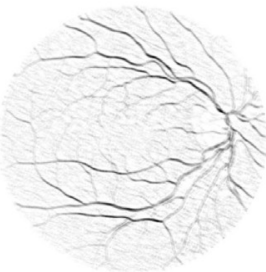

(h)

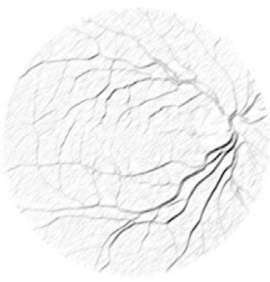

(m)

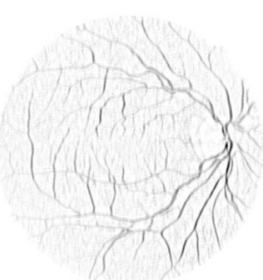

(d)

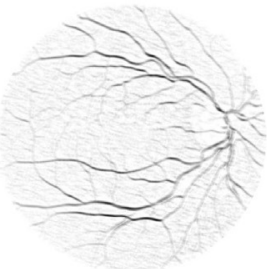

(i)

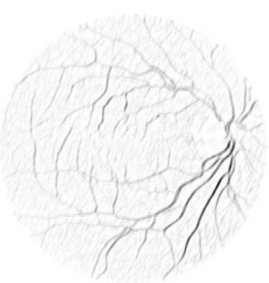

(n)

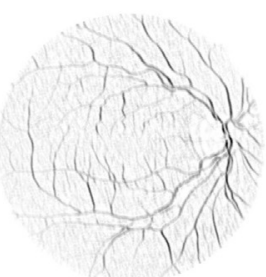

(e)

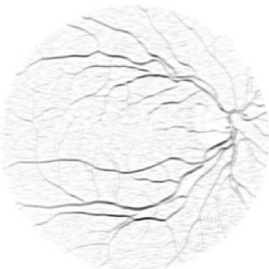

(j)

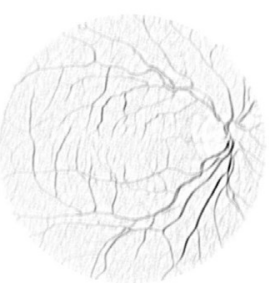

(o)

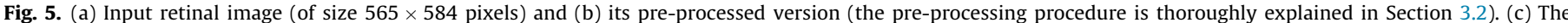

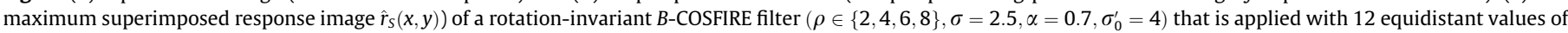

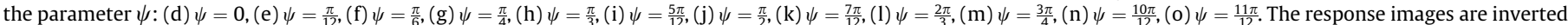
for clarity reasons.

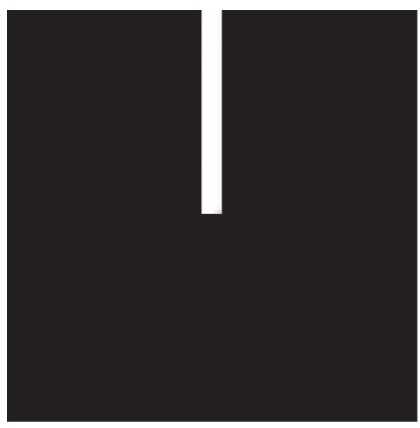

(a)

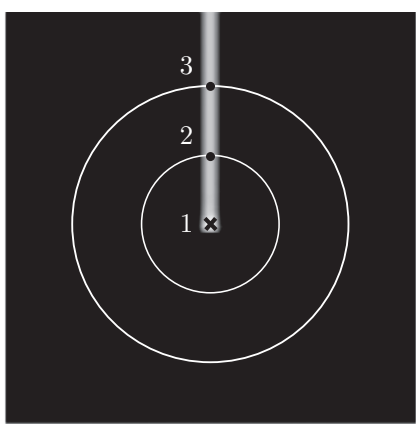

(b)

Fig. 6. (b) Example of the configuration of an asymmetric $B$-COSFIRE filter by a prototype bar-ending (a). The point of interest of the $B$-COSFIRE filter is indicated by the cross marker and lies on the end of the prototype line.

binary images with the ones that are manually segmented by the first observer as ground truth.

\subsection{Pre-processing}

For our experiments we only consider the green channel of retinal images. This decision is supported by previous works (Niemeijer et al., 2004; Staal et al., 2004; Mendonca and Campilho, 2006; Soares et al., 2006; Ricci and Perfetti, 2007) which found that the contrast between the vessels and the background is

better defined in the green channel. Conversely, the red channel has low contrast and the blue channel shows a small dynamic range. Mendonca and Campilho (2006) assessed the performance of different color representations such as the green component of the original RGB image, the luminance channel of the National Television Systems Committee (NTSC) color space and the $a^{*}$ component of the $L^{*} a^{*} b^{*}$ representation. They found that the highest contrast between vessels and background is, in general, shown in the green channel of the RGB image.

Due to the strong contrast around the FOV of the retinal images, the pixels close to the circumference might cause the detection of false vessels. Thus, we use the pre-processing algorithm proposed by Soares et al. (2006) to smoothen the strong contrast around the circular border of the FOV area. It uses a region of interest (ROI) determined by the FOV-mask of the retina. For the images in the DRIVE data set we use the provided FOV-mask images to determine the initial ROI. Since the STARE and the CHASE_DB1 data sets do not provide the FOV-mask images, we compute them by thresholding ${ }^{2}$ the luminosity plane of the $\mathrm{CIELab}^{3}$ version of the original RGB image.

Next, we dilate the border in the following iterative procedure. In the first iteration, we consider every black pixel that lies just on the exterior boundary of the FOV-mask. We then replace every such pixel with the mean value of the pixels of its 8-neighbours

\footnotetext{
${ }^{2}$ The thresholds are 0.5 and 0.1 for the STARE and CHASE_DB1 data sets, respectively.

${ }^{3}$ CIELab is a color space specified by the International Commission on Illumination.
} 


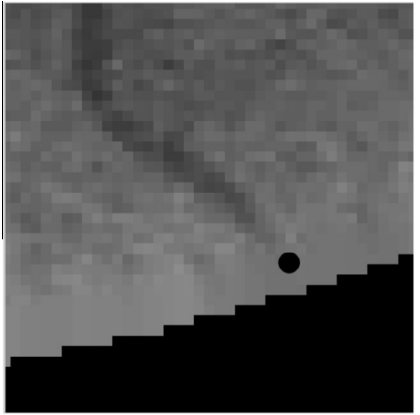

(a)

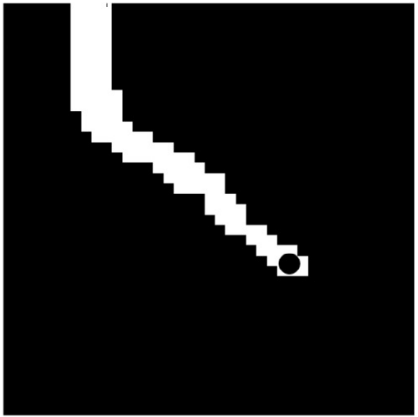

(b)

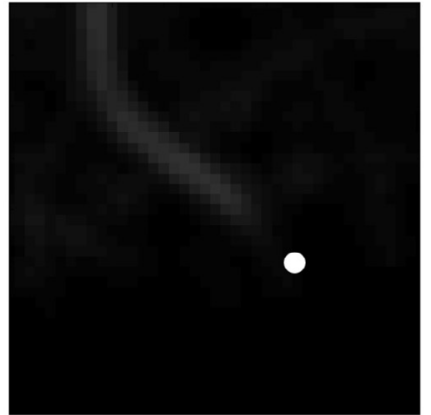

(c)

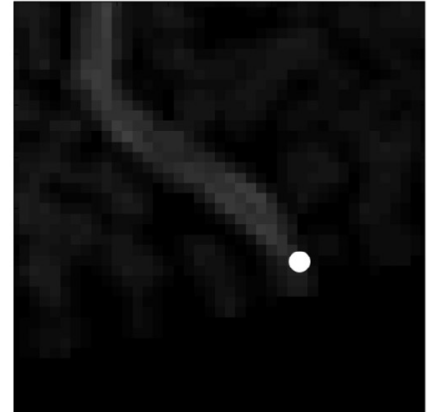

(d)

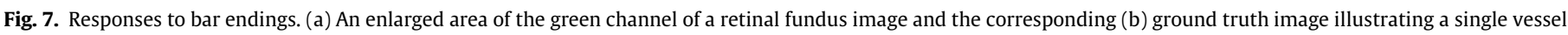

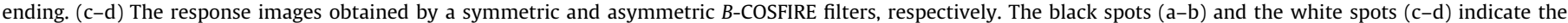
position of the vessel ending.

that are inside the ROI. After the first iteration, the radius of the ROI is increased by 1 pixel. We repeat this procedure 50 (in general $m$ ) times, as it is sufficient to avoid false detection of lines around the border of the FOV of the retina.

Finally, we enhance the image by using the contrast-limited adaptive histogram equalization (CLAHE) algorithm (Pizer et al., 1987). ${ }^{4}$ The CLAHE algorithm, which is commonly used as a pre-processing step in the analysis of retinal images (Fadzil et al., 2009; Setiawan et al., 2013), allows the improvement of the local contrast by avoiding the over-amplification of noise in relatively homogeneous regions. In Fig. 8 we illustrate all the pre-processing steps.

\subsection{Performance measurements}

For each test image we threshold the responses of a rotationinvariant $B$-COSFIRE filter by varying the values of parameter $t$ (in Eq. (6)) between 0 and 1 in steps of 0.01 . This threshold operation divides the pixels into two classes: vessels and non-vessels. Then, we compare every resulting binary image with the corresponding ground truth by computing the following four performance measurements: the pixels that belong to a vessel in the ground truth image and that are classified as vessels are counted as true positives (TP), otherwise they are counted as false negatives (FN). The pixels that belong to the background and that are classified as non-vessels, are counted as true negatives (TN), otherwise they are counted as false positives (FP).

In order to compare the performance of the proposed method with other state-of-the-art algorithms, we compute the accuracy (Acc), sensitivity (Se), specificity (Sp) and Matthews correlation coefficient (MCC). These metrics are defined as follows:

$$
\begin{aligned}
& A c c=\frac{T P+T N}{N}, S e=\frac{T P}{T P+F N}, S p=\frac{T N}{T N+F P}, \\
& M C C=\frac{T P / N-S \times P}{\sqrt{P \times S \times(1-S) \times(1-P)}},
\end{aligned}
$$

where $N=T N+T P+F N+F P, S=(T P+F N) / N$ and $P=(T P+F P) / N$.

The MCC is a measure of the quality of a binary classification. It is suitable even when the number of samples in the two classes varies substantially. As a matter of fact, this is the situation that we have at hand: the non-vessel pixels outnumber by seven times the vessel pixels. The MCC values vary between -1 and +1 . The higher the value the better the prediction is. A value of +1 indicates

\footnotetext{
${ }^{4}$ Other authors (Mendonca and Campilho, 2006; Marin et al., 2011) report that they pre-process the images with background homogenization algorithms based on wide Gaussian kernels, while others (Soares et al., 2006; Fraz et al., 2012) explicitly state that they do not employ any pre-processing step.
}

a perfect prediction, 0 indicates a prediction that is equivalent to random, and -1 indicates a completely wrong prediction.

Furthermore, for comparison purposes, we compute the receiving operator characteristics (ROC) curve, a method that is widely used to evaluate segmentation algorithms applied to retinal images. It is a tool that allows the analysis of the tradeoff between sensitivity and specificity. It is a 2D plot that illustrates the performance of a binary classifier system as its discrimination threshold is varied. For every threshold value we compute a $2 \mathrm{D}$ point on the ROC curve. The point represents the false positive rate $(F P R=1-S p)$ on the $x$-axis and the true positive rate $(T P R=S e)$ on the $y$-axis. The closer a ROC curve approaches the top-left corner the better the performance of the algorithm is. For a perfect classification a ROC curve has a point $(0,1)$. We consider the area under the ROC curve (AUC), which is equal to 1 for a perfect system, as a single measure to quantify the performance.

Since the pixels of the dark background outside the FOV area of the retina are easily detected, we consider only the pixels within the FOV area for the computation of the performance metrics.

\subsection{Results}

We carried out several experiments by considering different sets of parameters for the configuration and application of the $B$ COSFIRE filters. For the evaluation of the proposed method, we split each data set into an evaluation and test sub-sets. We used the evaluation set to determine the best parameters of the $B$-COSFIRE filters. For the DRIVE data set we used the training images for evaluation. The other two data sets, the STARE and the CHASE_DB1, contain only one set of images. For each of these two sets we used the first half of images for evaluation and, then we tested the proposed method on the entire data set.

First, we ran several experiments on the evaluation sets in order to determine the best parameters $\left(\rho, \sigma, \sigma_{0}, \alpha\right)$ of a symmetric $B$-COSFIRE filter by conducting a grid search and chose the set of parameters that gives the highest $M C C$ average value on the training images. We let the value of the parameter $\rho$ to increase by intervals of 2 pixels, that was empirically determined. In Table 1 we report the results that we achieved using a symmetric $B$-COSFIRE filter on the test sets along with the corresponding parameters that were determined in the evaluation phase.

Second, we trained an asymmetric $B$-COSFIRE filter on the evaluation sets in order to achieve stronger responses along the vessel endings. We observed that an asymmetric filter achieves higher response values in the proximity of the bar endings, but is less robust to noise than a symmetric $B$-COSFIRE filter. In Table 2 we report the results that we achieved using only the asymmetric 
(a)

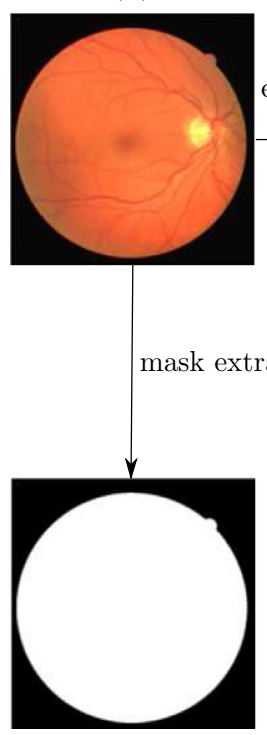

(e) (b)

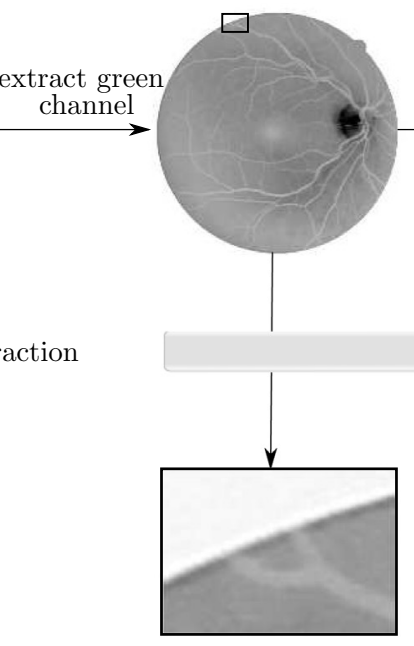

(f) (c)

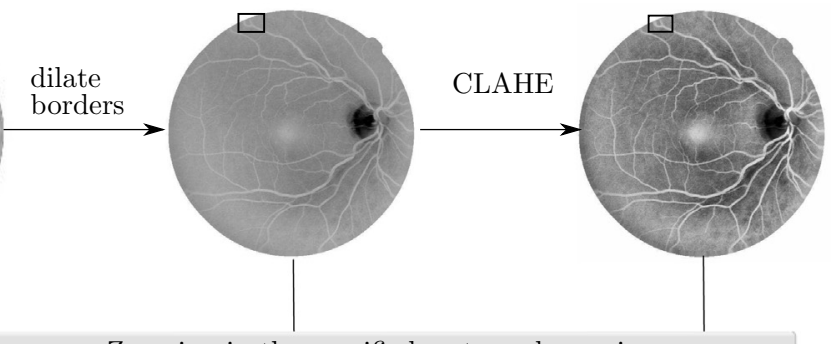

Zooming in the specified rectangular region

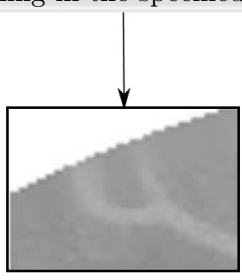

$(\mathrm{g})$

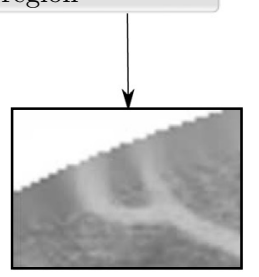

(h)

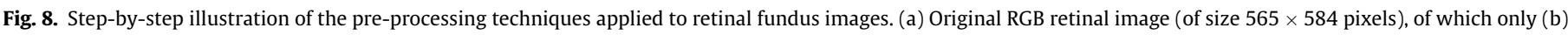

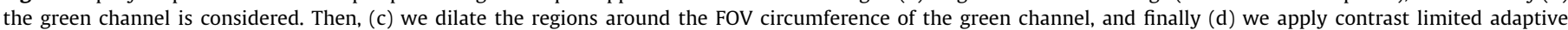

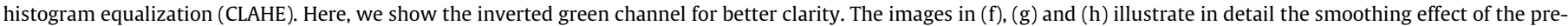

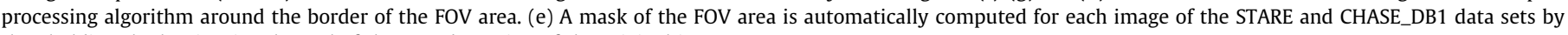
thresholding the luminosity channel of the CIELab version of the original image.

$B$-COSFIRE filter along with its configuration parameters for the three data sets.

Finally, we ran further experiments by summing the responses of symmetric and asymmetric $B$-COSFIRE filters. We determined a set of parameters for the asymmetric B-COSFIRE filter that best complement the ones of the symmetric $B$-COSFIRE filter. The addition of asymmetric $B$-COSFIRE filter contributes to a statistically significant improvement in the $M C C$ performance. This is confirmed by a right-tailed paired t-test statistic (DRIVE: $t(19)=3.4791, p<0.01$; STARE: $\left.t(19)=5.9276, p<10^{-5}\right)$. In Table 3 we report the results that we achieved by combining the responses of the two $B$-COSFIRE filters along with the corresponding sets of parameters.

It is worth noting that the value of $\sigma$ (the standard deviation of the outer Gaussian function of a DoG filter) is specific to each data set. Generally, as defined in (Petkov and Visser, 2005), the $\sigma$ value is

Table 1

(Top) Experimental results obtained by symmetric B-COSFIRE filters, one for each of the three data sets. (Bottom) The corresponding parameters that are automatically determined in an evaluation stage.

\begin{tabular}{|c|c|c|c|c|}
\hline \multicolumn{5}{|c|}{ Symmetric $B$-COSFIRE filter } \\
\hline & & DRIVE & STARE & CHASE_DB1 \\
\hline \multirow[t]{5}{*}{ Results } & Accuracy & 0.9427 & 0.9467 & 0.9411 \\
\hline & AUC & 0.9571 & 0.9487 & 0.9434 \\
\hline & Specificity & 0.9707 & 0.9689 & 0.9651 \\
\hline & Sensitivity & 0.7526 & 0.7543 & 0.7257 \\
\hline & MCC & 0.7395 & 0.7188 & 0.6791 \\
\hline \multirow[t]{4}{*}{ Parameters } & $\sigma$ & 2.4 & 2.7 & 4.8 \\
\hline & $\rho$ & $\{0,2,4, \ldots, 8\}$ & $\{0,2,4, \ldots, 12\}$ & $\{0,2,4, \ldots, 18\}$ \\
\hline & $\sigma_{0}$ & 3 & 1 & 3 \\
\hline & $\alpha$ & 0.7 & 0.6 & 0.2 \\
\hline
\end{tabular}

Table 2

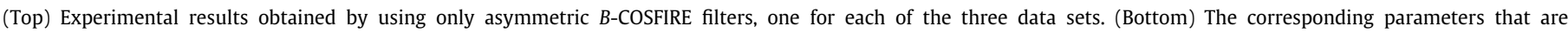
automatically determined in an evaluation stage.

\begin{tabular}{|c|c|c|c|c|}
\hline \multicolumn{5}{|c|}{ Asymmetric $B$-COSFIRE filter } \\
\hline & & DRIVE & STARE & CHASE_DB1 \\
\hline \multirow[t]{5}{*}{ Results } & Accuracy & 0.9422 & 0.9430 & 0.9270 \\
\hline & AUC & 0.9537 & 0.9536 & 0.9376 \\
\hline & Specificity & 0.9621 & 0.9742 & 0.9445 \\
\hline & Sensitivity & 0.7499 & 0.7765 & 0.7685 \\
\hline & MCC & 0.7369 & 0.7082 & 0.6433 \\
\hline \multirow[t]{4}{*}{ Parameters } & $\sigma$ & 1.9 & 2.4 & 4.7 \\
\hline & $\rho$ & $\{0,2,4, \ldots, 12\}$ & $\{0,2,4, \ldots, 22\}$ & $\{0,2,4, \ldots, 26\}$ \\
\hline & $\sigma_{0}$ & 2 & 1 & 2 \\
\hline & $\alpha$ & 0.1 & 0.1 & 0.1 \\
\hline
\end{tabular}


Table 3

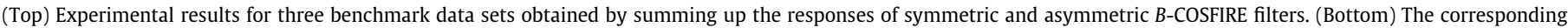
sets of parameter values.

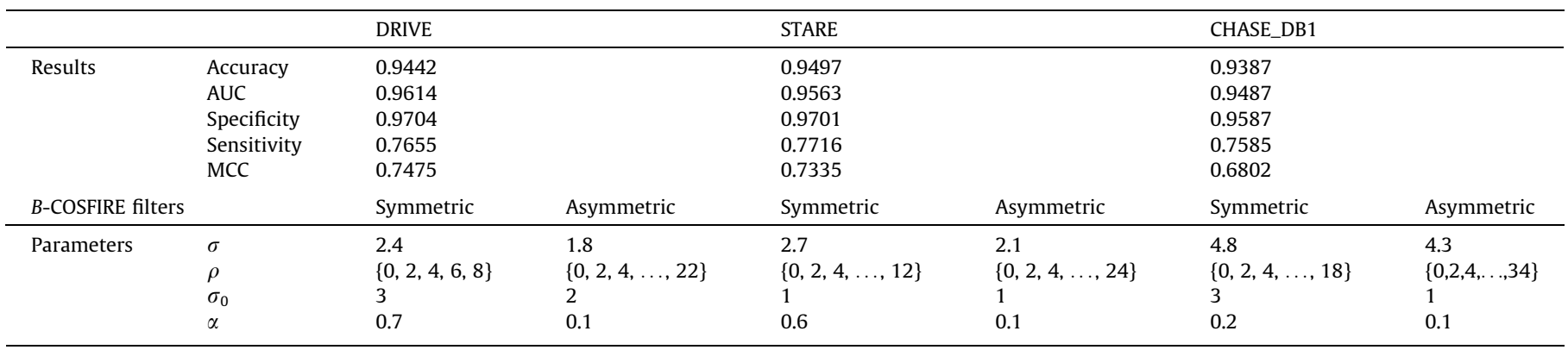

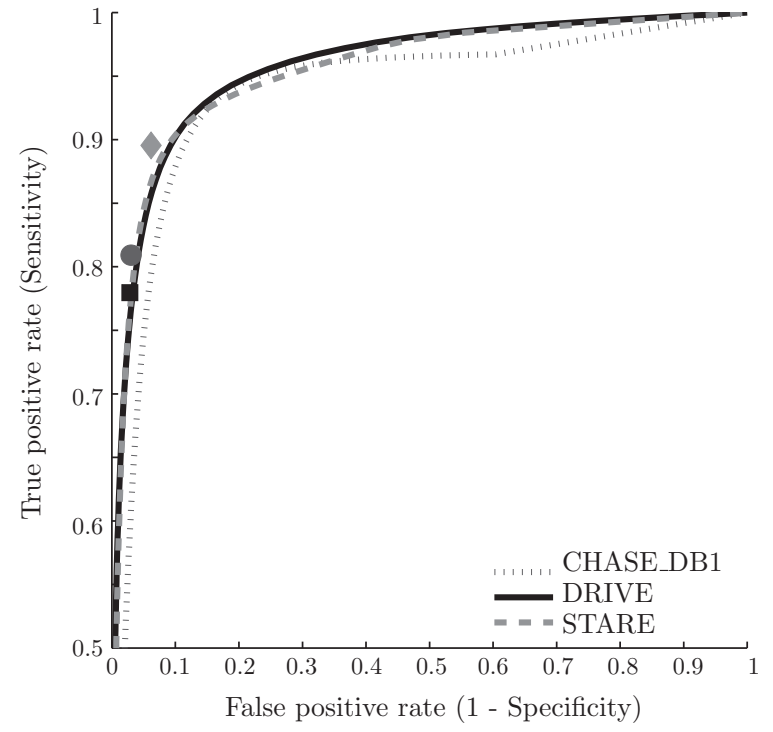

Fig. 9. ROC curves for DRIVE (solid line), STARE (dashed line) and CHASE_DB1 (dotted line) data sets. The results of the manual segmentation of the second observers are also reported on the graph. The squared marker ( $\boldsymbol{\square})$ is the performance of the second observer on the DRIVE data set, while the diamond $(\bullet)$ and the dot markers $(\bullet)$ are the results for the STARE and CHASE_DB1 data sets, respectively.

a function of the width $L$ of the vessel of interest: $\sigma=\frac{L}{2 \gamma} \sqrt{\left(1-\gamma^{2}\right) /(-\ln \gamma)}$, where $\gamma$ is a fraction of the $\sigma$ value (here we set $\gamma=0.5)$. The variation in the image resolutions of the three data sets requires the use of different $\sigma$ values. The fact that vessels are narrower at the end results in the configuration of an asymmetric filter with a $\sigma$ value smaller than that of the symmetric filter.

The sensitivity, specificity and accuracy values for each data set are obtained for a specific value of threshold $t$, the one which contributed to the maximum average MCC value of the corresponding data set. It is computed as follows. For a given test image and threshold $t$ we compute the MCC value. Subsequently, we compute the average of the MCC values of all the images to obtain a single performance measure denoted by $\overline{M C C}$. Several $\overline{M C C}$ values are obtained by varying the value of $t$ from 0 to 1 in steps of 0.01 . Finally, we choose the threshold value $t$ for a given data set that provides the maximum value of $\overline{M C C}$.

The ROC curves for the DRIVE, STARE and CHASE_DB1 data sets are depicted in Fig. 9. For reference purposes, we also show the segmentation results of the second observer when compared to what is considered the gold segmentation standard that is provided by the first observer.

The left column in Fig. 10 shows examples of retinal images, one for each of the three data sets, that are automatically obtained by

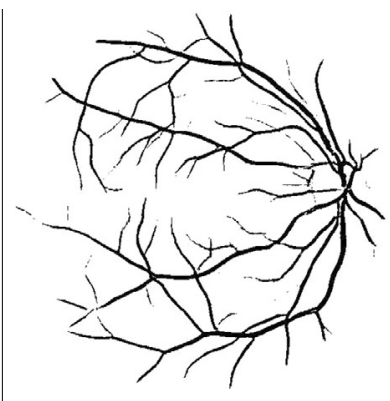

(a)

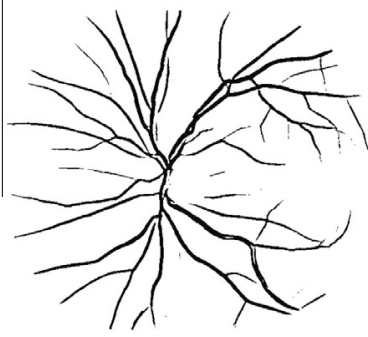

(b)

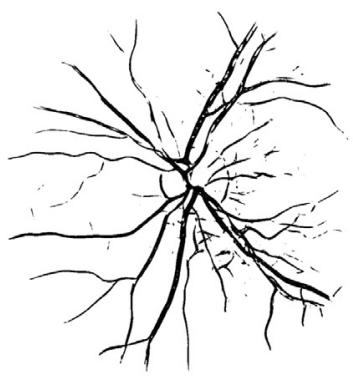

(c)

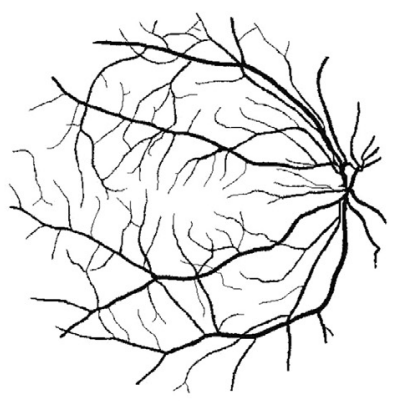

(d)

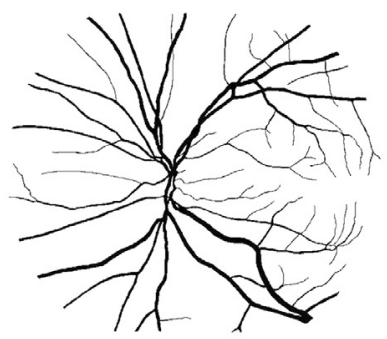

(e)

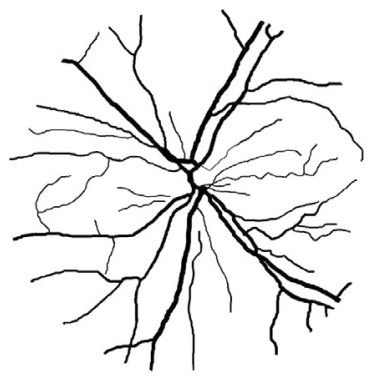

(f)
Fig. 10. Examples of segmented vessel trees that are automatically obtained by the proposed $B$-COSFIRE filter approach from images taken from the (a) DRIVE $(\mathrm{Se}=0.8746, \mathrm{Sp}=0.9662$ and $M C C=0.8005) \quad$ (b) STARE $\quad(\mathrm{Se}=0.8575$, $S p=0.9754$ and $M C C=0.8097)$ and (c) CHASE_DB1 ( $S e=0.8360, S p=0.9548$ and $M C C=0.7180$ ) data sets. Images in (d), (e) and (f) show the corresponding manually segmented images by the first observer.

the proposed approach. The right column shows the corresponding ground truth images that were manually segmented by the first observer of each data set. 


\section{Discussion}

The method that we propose is a trainable filter approach that we apply in an unsupervised way. The performance results that we achieve on the DRIVE, STARE, and CHASE_DB1 benchmark data sets are better than many state-of-the-art unsupervised and supervised algorithms (Tables 4-6). We evaluate the performance of the proposed method using the MCC value because it is a measure of the quality of a binary classification that is suitable when the samples in the two classes are unbalanced. For the sake of comparison, we move along the ROC curves in order to evaluate the performance of the B-COSFIRE approach with respect to the best results achieved by other unsupervised methods. For the DRIVE data set and for the same specificity $(S p=0.9764)$ reported by Mendonca and Campilho (2006) we achieve a sensitivity of 0.7376 , which is marginally better. Similarly, for the STARE data set and for the same specificity reported by Mendonca and Campilho (2006) and Al-Diri et al. (2009) $(S p=0.9730$ and $S p=0.9681)$ we achieve a sensitivity of 0.7554 and 0.7848 respectively, which is a significantly better result. We also achieve the best AUC value for the DRIVE data set with respect to all other unsupervised approaches. As to the CHASE_DB1 data set, there are no other state-of-the-art unsupervised approaches to which we can compare.

While our method is unsupervised and it is more appropriate to compare it to other unsupervised methods, here we observe that its performance is comparable to some supervised methods or slightly lower to others. Supervised methods are based on machine learning techniques and typically require high-dimensional pixel-wise feature vectors to train a classifier that discriminates vessel from non-vessel pixels. For instance, Fraz et al. (2012) characterize every pixel by a feature vector of nine elements, obtained by various filtering and morphological operations. Time complexity grows with increasing number of dimensions as more operations need to be performed. In our approach we characterize every pixel with only two values, one from a vessel-selective filter and one from a vessel-ending-selective filter and combine them by summation. This results in a very efficient way of processing a retinal image. It achieves performance results that are close to the supervised methods, which require high-dimensional feature vectors followed by an extensive training algorithm to learn the best separation margin in the feature space. For the DRIVE data set

\section{Table 6}

Performance results of the proposed unsupervised (unsup.) B-COSFIRE filter approach on CHASE_DB1 data set compared to the only one other existing supervised (sup.) method. The performance results achieved by the proposed method are reported in bold.

\begin{tabular}{llllll}
\hline \multicolumn{6}{l}{ CHASE_DB1 } \\
\cline { 3 - 5 } & Method & Se & Sp & AUC & Acc \\
\hline Unsup. & B-COSFIRE & $\mathbf{0 . 7 5 8 5}$ & $\mathbf{0 . 9 5 8 7}$ & $\mathbf{0 . 9 4 8 7}$ & $\mathbf{0 . 9 3 8 7}$ \\
Sup. & Fraz et al. (2012) & 0.7224 & 0.9711 & 0.9712 & 0.9469 \\
\hline
\end{tabular}

Table 4

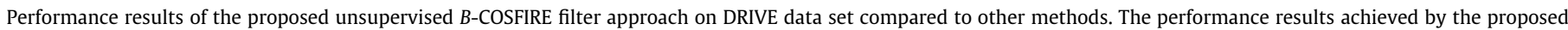
method are reported in bold.

\begin{tabular}{|c|c|c|c|c|c|}
\hline DRIVE & & & & & \\
\hline & Method & Se & Sp & AUC & Acc \\
\hline \multirow[t]{9}{*}{ Unsupervised } & B-COSFIRE & 0.7655 & 0.9704 & 0.9614 & 0.9442 \\
\hline & Chauduri et al. (1989) & - & - & 0.7878 & 0.8773 \\
\hline & Mendonca and Campilho (2006) & 0.7344 & 0.9764 & - & 0.9463 \\
\hline & Martinez-Pérez et al. (2007) & 0.7246 & 0.9655 & - & 0.9344 \\
\hline & Al-Rawi et al. (2007) & - & - & 0.9435 & 0.9535 \\
\hline & Ricci and Perfetti (2007) & - & - & 0.9558 & 0.9563 \\
\hline & Al-Diri et al. (2009) & 0.7282 & 0.9551 & - & - \\
\hline & Cinsdikici and Aydin (2009) & - & - & 0.9407 & 0.9293 \\
\hline & Lam et al. (2010) & - & - & 0.9614 & 0.9472 \\
\hline \multirow[t]{6}{*}{ Supervised } & Niemeijer et al. (2004) & - & - & 0.9294 & 0.9416 \\
\hline & Staal et al. (2004) & - & - & 0.9520 & 0.9441 \\
\hline & Soares et al. (2006) & 0.7332 & 0.9782 & 0.9614 & 0.9466 \\
\hline & Ricci and Perfetti (2007) & - & - & 0.9633 & 0.9595 \\
\hline & Marin et al. (2011) & 0.7067 & 0.9801 & 0.9588 & 0.9452 \\
\hline & Fraz et al. (2012) & 0.7406 & 0.9807 & 0.9747 & 0.9480 \\
\hline
\end{tabular}

Table 5

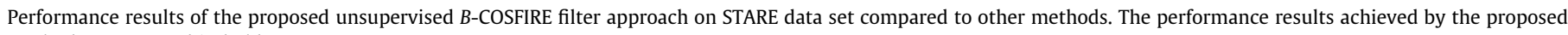
method are reported in bold.

\begin{tabular}{|c|c|c|c|c|c|}
\hline \multicolumn{6}{|l|}{ STARE } \\
\hline & Method & Se & Sp & AUC & Acc \\
\hline \multirow[t]{9}{*}{ Unsupervised } & B-COSFIRE & 0.7716 & 0.9701 & 0.9563 & 0.9497 \\
\hline & Hoover et al. (2000) & 0.6747 & 0.9565 & 0.7590 & 0.9275 \\
\hline & Jiang and Mojon (2003) & - & - & 0.9298 & 0.9009 \\
\hline & Mendonca and Campilho (2006) & 0.6996 & 0.9730 & - & 0.9479 \\
\hline & Martinez-Pérez et al. (2007) & 0.7506 & 0.9569 & - & 0.9410 \\
\hline & Al-Rawi et al. (2007) & - & - & 0.9467 & 0.9090 \\
\hline & Ricci and Perfetti (2007) & - & - & 0.9602 & 0.9584 \\
\hline & Al-Diri et al. (2009) & 0.7521 & 0.9681 & - & - \\
\hline & Lam et al. (2010) & - & - & 0.9739 & 0.9567 \\
\hline \multirow[t]{5}{*}{ Supervised } & Staal et al. (2004) & - & - & 0.9614 & 0.9516 \\
\hline & Soares et al. (2006) & 0.7207 & 0.9747 & 0.9671 & 0.9480 \\
\hline & Ricci and Perfetti (2007) & - & - & 0.9680 & 0.9646 \\
\hline & Marin et al. (2011) & 0.6944 & 0.9819 & 0.9769 & 0.9526 \\
\hline & Fraz et al. (2012) & 0.7548 & 0.9763 & 0.9768 & 0.9534 \\
\hline
\end{tabular}


Table 7

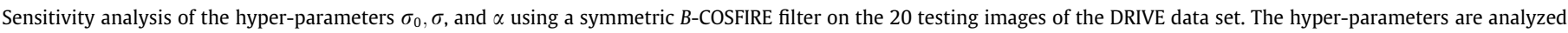

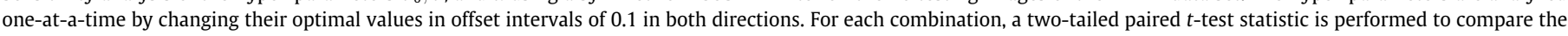
resulting $20 \mathrm{MCC}$ values with those obtained by the optimal parameters (i.e. offset of 0 ). The $t$-values rendered in bold indicate statistical significance with $p<0.05$.

\begin{tabular}{|c|c|c|c|c|c|c|c|c|c|c|c|c|}
\hline & Offset & -0.5 & -0.4 & -0.3 & -0.2 & -0.1 & 0 & 0.1 & 0.2 & 0.3 & 0.4 & 0.5 \\
\hline$\sigma_{0}$ & $\boldsymbol{t}(\mathbf{1 9})$ & -0.34 & -0.06 & 0.26 & 0.30 & 0.33 & - & -0.35 & -0.96 & -1.36 & -1.68 & -1.87 \\
\hline$\sigma$ & $\boldsymbol{t}(\mathbf{1 9})$ & -2.24 & -1.94 & -1.42 & -0.84 & -0.43 & - & -0.24 & -0.63 & -1.26 & -1.66 & -2.35 \\
\hline$\alpha$ & $\boldsymbol{t}(\mathbf{1 9})$ & -4.71 & -3.28 & -2.41 & -1.34 & -0.88 & - & -0.07 & -0.78 & -1.55 & -1.80 & -2.72 \\
\hline
\end{tabular}

and for the same specificity reported by Marin et al. (2011) $(S p=0.9801)$ we achieve a sensitivity of 0.7040 , which is comparable with the one $(0.7067)$ that they report. For the STARE data set and for the same specificity reported by Marin et al. (2011) and Fraz et al. (2012) we achieve comparable sensitivity of 0.6908 and 0.7393 , respectively. The ROC curves in Fig. 9 indicate that the performance of our method on the DRIVE and STARE data sets is comparable to that of the second human observer. For the CHASE_DB1 data set, however, the second human observer achieves a performance that is better than that of our method.

The $B$-COSFIRE filter that we propose is versatile as its selectivity is not predefined in the implementation but rather it is determined from a given prototype pattern in an automatic configuration process. As a matter of fact, here we demonstrated the configuration of two kinds of $B$-COSFIRE filters, namely symmetric and asymmetric, that give strong responses along vessels and at vessel endings, respectively. The configuration of these two types of filters was achieved by using two different prototype patterns. In both cases we used prototype bar patterns of constant width and consequently the filters resulted in sets of tuples with the same value of parameter $\sigma$. One may, however, use tapered bar structures as prototype patterns which would result in tuples with different $\sigma$ values. In principle, one can also configure $B$-COSFIRE filters that are selective for more complicated structures, such as bifurcations or crossovers. The B-COSFIRE filters used in this work are selective for elongated structures and achieve strong responses along the vessels and their endings but show slightly lower responses for bifurcations and crossovers. One may configure other COSFIRE filters selective for various types of bifurcations and crossovers and combine their responses with those of the vessel and vessel-ending-selective filters that we propose.

The B-COSFIRE filters can also be employed to detect vessels in other medical images, such as mammography and computed tomography. The proposed segmentation approach may also be used in non-medical applications that contain vessel-like structures, such as palmprints segmentation for biometric systems (Kong et al., 2009).

We aim to extend this work in various aspects. One direction for further investigation will focus on the configuration of a set of COSFIRE filters selective for different patterns, such as vessels, bifurcations and crossovers at different space-scales. The responses of such filters can be used to form a pixel-wise descriptor and then to train a supervised classifier in order to discriminate vessel from non-vessel pixels. This will allow us to perform thorough analysis with respect to tiny vessels, vessels around the optic disc, and differentiation of vessels from abnormalities. Another direction for future work is to consider the depth dimension and configure COSFIRE filters for 3D vessel structures that can be applied, for instance, to detect the blood vessels in angiography images of the brain.

The configuration and application of a B-COSFIRE filter is conceptually simple and easy to implement: it involves convolution with DoG filters, blurring the DoG responses, shifting the blurred responses towards the center of the concerned $B$-COSFIRE filter and computing a point-wise weighted geometric mean. The center-on DoG filters that we used in this work are not intrinsic to the method. Elsewhere we demonstrated that COSFIRE filters can be configured by Gabor filters for the detection of patterns
Table 8

Comparative analysis of the methods in terms of time required to process a single image from the DRIVE and STARE data sets.

\begin{tabular}{ll}
\hline Method & Processing time \\
\hline B-COSFIRE & $\mathbf{1 0 ~ s}$ \\
Jiang and Mojon (2003) & $20 \mathrm{~s}$ \\
Staal et al. (2004) & $15 \mathrm{~min}$ \\
Mendonca and Campilho (2006) & $2.5 \mathrm{~min}$ \\
Soares et al. (2006) & $3 \mathrm{~min}$ \\
Al-Diri et al. (2009) & $11 \mathrm{~min}$ \\
Lam et al. (2010) & $13 \mathrm{~min}$ \\
Marin et al. (2011) & $1.5 \mathrm{~min}$ \\
Fraz et al. (2012) & $2 \mathrm{~min}$ \\
\hline
\end{tabular}

characterized by multiple lines/edges of different orientations (Azzopardi and Petkov, 2013b). In particular, Azzopardi and Petkov (2013a) demonstrated that COSFIRE filters can be used to detect vascular bifurcations in retinal fundus images. Moreover, we show that by using a collection of center-off and center-on DoG filters we can effectively configure a contour operator (Azzopardi and Petkov, 2012).

The performance of a $B$-COSFIRE filter is affected by the values of the parameters $\left(\sigma_{0}, \sigma, \alpha\right)$ that are automatically selected in the configuration stage. In Table 7 we report one-at-a-time sensitivity analysis, which shows that the parameter $\sigma_{0}$ is the least sensitive, followed by $\sigma$ and then by $\alpha$.

The application of a rotation-invariant $B$-COSFIRE filter is very efficient. A B-COSFIRE filter is defined as a set of 3-tuples and each tuple requires the blurring and shifting of a DoG filter response in a certain position. The computation of one blurred and shifted response (for the same values of $\sigma$ and $\rho$ ), for instance with $s_{\sigma, \rho, \phi=0}(x, y)$ is sufficient. The result of $s_{\sigma, \rho, \phi}(x, y)$ for any value of $\phi$ can be obtained from the result of the output of $s_{\sigma, \rho, \phi=0}(x, y)$ by appropriate shifting. Therefore, the number of computations required depends on the number of unique combinations of the values of parameters $\sigma$ and $\rho$. In practice, the Matlab implementation $^{5}$ that we used for our experiments takes less than $10 \mathrm{~s}$ to process each image from the DRIVE $(565 \times 584$ pixels $)$ and STARE $(700 \times 605$ pixels) data sets and less than $25 \mathrm{~s}$ to process an image from the CHASE_DB1 data set $(1280 \times 960$ pixels $)$, on a personal computer equipped with a $2 \mathrm{GHz}$ processor. The duration times that we report include the processing of both symmetric and asymmetric $B$-COSFIRE filters to a single image. Note that the implementation that we use here is based on sequential processing. The B-COSFIRE approach can, however, be implemented in parallel mode such that the blurring and shifting operations for different pairs of $(\sigma, \rho)$ can be processed on multiple processors, simultaneously. It is worth noting that on images taken from the DRIVE and STARE data sets our implementation is significantly less time-consuming than other approaches tested on similar hardware, as reported in Table 8.

The proposed $B$-COSFIRE approach differs mainly in the following three aspects from other unsupervised approaches. First, a $B$ COSFIRE filter is trainable as its selectivity is not predefined in the implementation but rather it is determined from a given prototype pattern in an automatic configuration process. Other methods

\footnotetext{
5 The Matlab implementation is available at http://matlabserver.cs.rug.nl/.
} 


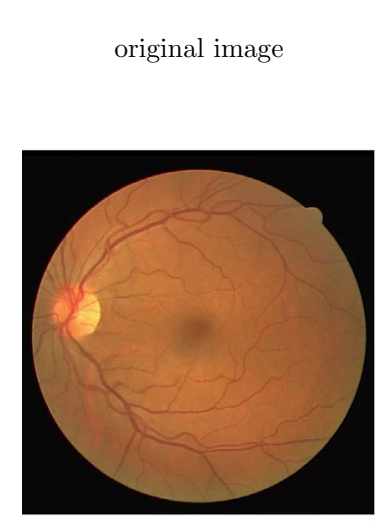

(a)

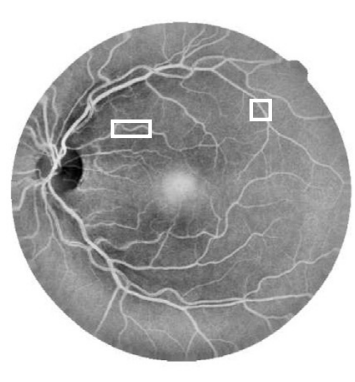

(b)

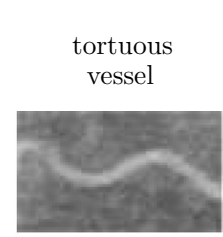

(c)

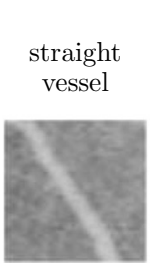

(f)

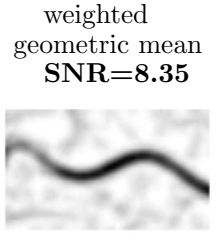

(d)

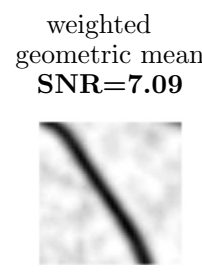

(g) weighted

arithmetic mean

$\mathrm{SNR}=7.02$

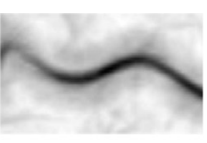

(e)

weighted

arithmetic mean

$\mathrm{SNR}=6.47$

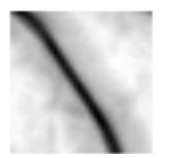

(h)

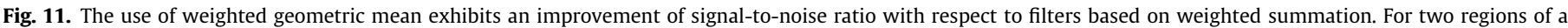

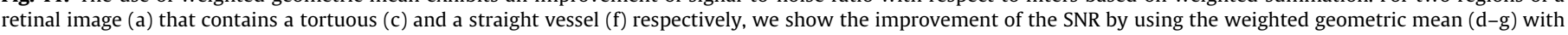
respect to the weighted summation $(\mathrm{e}-\mathrm{h})$.

use a predefined set of kernels to filter the input image. Second, a $B$-COSFIRE filter achieves rotation-invariance in a very efficient way. It only requires the appropriate shifting of the blurred DoG responses followed by weighted geometric mean. On the contrary, other methods (Chauduri et al., 1989; Hoover et al., 2000; Al-Rawi et al., 2007; Ricci and Perfetti, 2007) achieve rotation invariance by convolving the input image with rotated versions of the original kernel. For those methods the number of convolutions is dependent on the number of orientations for which the operator is applied. Third, the weighted geometric mean that we use is a nonlinear function which produces a response only when all the filter defining points of interest are present. This type of function is more robust to noise than other methods (Hoover et al., 2000; Ricci and Perfetti, 2007) that rely on weighted summations (convolutions).

In Fig. 11, we show how the response of a B-COSFIRE filter that is computed as the weighted geometric mean of the afferent DoG responses is less affected by noise in comparison to the response of a $B$-COSFIRE filter with the same support structure but that uses weighted arithmetic mean. We compute the signal-to-noise ratio as $S N R=\mu_{s i g} / \sigma_{b g}$, where $\mu_{s i g}$ and $\sigma_{b g}$ are the average signal value and the background standard deviation, respectively. For a rather straight vessel (Fig. 11f) and using weighted geometric mean the filter achieves a SNR of 8.35, which is higher than that achieved by using weighted arithmetic mean $(S N R=7.02)$. The difference is observed also for highly tortuous vessels (Fig. 11c), for which a SNR of 7.09 is achieved by the weighted geometric mean which is again higher than its counterpart $(S N R=6.47)$.

\section{Conclusions}

The results that we achieve on three benchmark data sets, DRIVE ( $\mathrm{Se}=0.7655, \mathrm{Sp}=0.9704)$, STARE $(\mathrm{Se}=0.7716, \mathrm{Sp}=0.9701$ ) and CHASE_DB1 $(\mathrm{Se}=0.7585, \mathrm{Sp}=0.9587$ ) are higher than many of the state-of-the-art methods. The high effectiveness achieved by the approach that we propose is coupled with high efficiency. In fact, the proposed method is the most time-efficient algorithm for blood vessels segmentation in retinal fundus images published so far.

The B-COSFIRE filter is versatile as it can be configured, in an automatic process, to detect any given vessel-like patterns. As its response is achieved by computing the weighted geometric mean of the responses of DoG filters with collinearly aligned supports. The B-COSFIRE filter shows higher robustness to noise than methods based on weighted summation or convolution (template matching). Besides vessel segmentation it can also be used to detect features of interest, such as vascular bifurcations and crossovers.

\section{References}

Abramoff, M.D., Garvin, M.K., Sonka, M., 2010. Retinal imaging and image analysis. Al-Diri, B., Hunter, A., Steel, D., Habib, M., Hudaib, T., Berry, S., 2008. Review - a reference data set for retinal vessel profiles, in: EMBS 2008. 30th Annual International Conference of the IEEE Engineering in Medicine and Biology Society, 2008, pp. 2262-2265.

Al-Diri, B., Hunter, A., Steel, D., 2009. An active contour model for segmenting and measuring retinal vessels. IEEE Trans. Med. Imag. 28, 1488-1497.

Al-Rawi, M., Qutaishat, M., Arrar, M., 2007. An improved matched filter for blood vessel detection of digital retinal images. Comput. Biol. Med. 37, 262-267.

Azzopardi, G., Petkov, N., 2012. A CORF computational model of a simple cell that relies on LGN input outperforms the Gabor function model. Biol. Cybern. 106, $177-189$.

Azzopardi, G., Petkov, N., 2013a. Automatic detection of vascular bifurcations in segmented retinal images using trainable COSFIRE filters. Pattern Recogn. Lett. 34, 922-933.

Azzopardi, G., Petkov, N., 2013b. Trainable COSFIRE filters for keypoint detection and pattern recognition. IEEE Trans. Pattern Anal. Mach. Intell. 35, 490-503.

Chauduri, S., Chatterjee, S., Katz, N., Nelson, M., Goldbaum, M., 1989. Detection of blood-vessels in retinal images using two-dimensional matched-filters. IEEE Trans. Med. Imag. 8, 263-269.

Chutatape, O., Liu Zheng, Krishnan, S., 1998. Retinal blood vessel detection and tracking by matched Gaussian and Kalman filters, in: Chang, H., Zhang, Y. (Eds.), Proc. 20th Annu. Int. Conf. IEEE Eng. Med. Biol. Soc. (EMBS’98), pp. 3144-3149.

Cinsdikici, M.G., Aydin, D., 2009. Detection of blood vessels in ophthalmoscope images using MF/ant (matched filter/ant colony) algorithm. Comput. Methods Prog. Biomed., 85-95.

Fadzil, M., Nugroho, H., Nugroho, H., Iznita, I., 2009. Contrast enhancement of retinal vasculature in digital fundus image, in: 2009 International Conference on Digital Image Processing, pp. 137-141.

Fang, B., Hsu, W., Lee, M., 2003. Reconstruction of vascular structures in retinal images. Proceedings 2003 International Conference on Image Processing (Cat. No.03CH37429), vol. 3. IEEE Signal Process. Soc., pp. II-157-II-160.

Fraz, M., Remagnino, P., Hoppe, A., Uyyanonvara, B., Rudnicka, A., Owen, C., Barman, S., 2012. An ensemble classification-based approach applied to retinal blood vessel segmentation. IEEE Trans. Biomed. Eng. 59, 2538-2548.

Gang, L., Chutatape, O., Krishnan, S., 2002. Detection and measurement of retinal vessels, in fundus images using amplitude modified second-order Gaussian filter. IEEE Trans. Biomed. Eng. 49, 168-172.

Grisan, E., Foracchia, M., Ruggeri, A., 2008. A novel method for the automatic grading of retinal vessel tortuosity. IEEE Trans. Med. Imag. 27, 310-319.

Harding, S., Broadbent, D., Neoh, C. White, M., Vora, J., 1995. Sensitivity and specificity of photography and direct ophthalmoscopy in screening for sight threatening eye disease - the Liverpool diabetic eye study. Brit. Med. J. 311 $1131-1135$.

Heneghan, C., Flynn, J., O'Keefe, M., Cahill, M., 2002. Characterization of changes in blood vessel width and tortuosity in retinopathy of prematurity using image analysis. Med. Image Anal. 6, 407-429. 
Hoover, A., Kouznetsova, V., Goldbaum, M., 2000. Locating blood vessels in retinal images by piecewise threshold probing of a matched filter response. IEEE Trans. Med. Imag. 19, 203-210.

Irvine, G., Casagrande, V., Norton, T., 1993. Center surround relationships of magnocellular, parvocellular, and koniocellular relay cells in primate lateral geniculate-nucleus. Visual Neurosci. 10, 363-373.

Jiang, X., Mojon, D., 2003. Adaptive local thresholding by verification-based multithreshold probing with application to vessel detection in retinal images. IEEE Trans. Pattern Anal. Mach. Intell. 25, 131-137.

Kong, A., Zhang, D., Kamel, M., 2009. A survey of palmprint recognition. Pattern Recogn. 42, 1408-1418.

Lam, B., Gao, Y., Liew, A.C., 2010. General retinal vessel segmentation using regularization-based multiconcavity modeling. IEEE Trans. Med. Imag. 29, 1369-1381.

Liu, I., Sun, Y., 1993. Recursive tracking of vascular networks in angiograms based on the detection deletion scheme. IEEE Trans. Med. Imag. 12, 334-341.

Marin, D., Aquino, A., Emilio Gegundez-Arias, M., Manuel Bravo, J., 2011. A new supervised method for blood vessel segmentation in retinal images by using gray-level and moment invariants-based features. IEEE Trans. Med. Imag. 30, $146-158$.

Martinez-Pérez, M.E., Hughes, A.D., Thom, S.A., Bharath, A.A., Parker, K.H., 2007. Segmentation of blood vessels from red-free and fluorescein retinal images. Med. Image Anal. 11, 47-61.

Mendonca, A.M., Campilho, A., 2006. Segmentation of retinal blood vessels by combining the detection of centerlines and morphological reconstruction. IEEE Trans. Med. Imag. 25, 1200-1213.

Niemeijer, M., Staal, J., van Ginneken, B., Loog, M., Abramoff, M., 2004. Comparative study of retinal vessel segmentation methods on a new publicly available database, in: Proc. of the SPIE - The International Society for Optical Engineering, pp. 648-656 (Medical Imaging 2004. Image Processing, 16-19 February 2004, San Diego, CA, USA).

Owen, C.G., Rudnicka, A.R., Mullen, R., Barman, S.A., Monekosso, D., Whincup, P.H. $\mathrm{Ng}$, J., Paterson, C., 2009. Measuring retinal vessel tortuosity in 10-year-old children: validation of the computer-assisted image analysis of the retina (CAIAR) program. Invest. Ophthalmol. Vis. Sci. 50, 2004-2010.
Petkov, N., Visser, W.T., 2005. Modifications of Center-Surround, Spot Detection and Dot-Pattern Selective operators. Technical Report CS 2005-9-01. Institute of Mathematics and Computing Science, University of Groningen, The Netherlands.

Pizer, S., Amburn, E., Austin, J., Cromartie, R., Geselowitz, A., Greer, T., Ter Haar Romeny, B., Zimmerman, J., Zuiderveld, K., 1987. Adaptative histogram equalization and its varations. Comput. Vis. Graph. Image Process. 39, 355368.

Ricci, E., Perfetti, R., 2007. Retinal blood vessel segmentation using line operators and support vector classification. IEEE Trans. Med. Imag. 26, 1357-1365.

Rodieck, R.W., 1965. Quantitative analysis of cat retinal ganglion cell response to visual stimuli. Vis. Res. 5, 583-601.

Setiawan, A., Mengko, T., Santoso, O., Suksmono, A., 2013. Color retinal image enhancement using clahe. In: 2013 International Conference on ICT for Smart Society (ICISS), pp. 1-3.

Soares, J.V.B., Leandro, J.J.G., Cesar Jr., R.M., Jelinek, H.F., Cree, M.J., 2006. Retinal vessel segmentation using the 2-D Gabor wavelet and supervised classification. IEEE Trans. Med. Imag. 25, 1214-1222.

Staal, J., Abramoff, M., Niemeijer, M., Viergever, M., van Ginneken, B., 2004. Ridgebased vessel segmentation in color images of the retina. IEEE Trans. Med. Imag. 23, 501-509.

Tolias, Y., Panas, S., 1998. A fuzzy vessel tracking algorithm for retinal images based on fuzzy clustering. IEEE Trans. Med. Imag. 17, 263-273.

von Wendt, G., Heikkila, K., Summanen, P., 1999. Assessment of diabetic retinopathy using two-field $60^{\circ}$ fundus photography. A comparison between red-free, blackand-white prints and colour transparencies. Acta Ophthalmol. Scand. 77, 638647.

Xu, X., Bonds, A., Casagrande, V., 2002. Modeling receptive-field structure of koniocellular, magnocellular, and parvocellular LGN cells in the owl monkey (Aotus trivigatus). Visual Neurosci. 19, 703-711.

Zana, F., Klein, J., 2001. Segmentation of vessel-like patterns using mathematical morphology and curvature evaluation. IEEE Trans. Med. Imag. 10, 1010-1019.

Zhou, L., Rzeszotarski, M., Singerman, L., Chokreff, J., 1994. The detection and quantification of retinopathy using digital angiograms. IEEE Trans. Med. Imag. 13, 619-626. 